\section{Impact of cataract surgery on vision- related life performances: the usefulness of Real-Life Vision Test for cataract surgery outcomes evaluation}

\author{
Abstract \\ Purpose Real-Life Vision Test (RLVT) is a \\ newly developed performance-based \\ measures of functional vision. This present \\ study is designed to determine whether it \\ could be a meaningful assessment for cataract \\ surgery outcomes evaluation. \\ Patients and methods Age-related cataract \\ patients (56) who scheduled for bilateral \\ cataract surgery and 44 age-matched controls \\ were evaluated by four types of \\ measurements: (1) demographic, medical, \\ cognitive and depressive evaluation, and the \\ reaction time testing; (2) clinical measures \\ (visual acuity, contrast sensitivity, stereopsis, \\ and color perception); (3) the 25-item National \\ Eye Institute's Visual Functioning \\ Questionnaire (NEI-VFQ); (4) the RLVT. \\ Spearman's coefficients and multiple \\ regression analysis were conducted to \\ investigate the relationship among RLVT, \\ clinical measures, and self-report assessment \\ of visual function. \\ Results The results of RLVT, clinical \\ measures, and NEI-VFQ total scores were \\ improved significantly after cataract surgery. \\ There were no differences between control \\ subjects and post-surgery patients with \\ respect to NEI-VFQ-25 total scores, self-rating \\ depression scale scores and three tasks of \\ RLVT. Change of RLVT was significantly \\ associated with the change of clinical \\ measures in the cataract group. Multiple \\ regression analysis demonstrated that change \\ of distance, intermediate, and near visual \\ acuity, and binocular contrast sensitivity were \\ significant predictors of improvements \\ of RLVT.
}

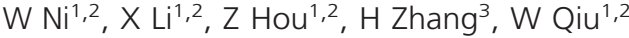
and $W$ Wang ${ }^{1,2}$
Conclusions Cataract surgery could improve real-world visual ability effectively for cataract patients. Our study highlights the potential usefulness of RLVT as an adjunct to the current outcomes evaluation system for cataract surgery. The use of RLVT combined with clinical and self-survey methods may be the comprehensive strategy to manifest the impact of cataract surgery on patients' overall vision-related quality of life.

Eye (2015) 29, 1545-1554; doi:10.1038/eye.2015.147; published online 14 August 2015

\section{Introduction}

Age-related cataract is a major public health issue globally and significant cause of visual impairment. ${ }^{1}$ Currently, cataract surgery is the most common undertaken surgical intervention in healthcare. ${ }^{2}$ The ultimate goal of cataract surgery is to improve the patient's visual function and, eventually, their quality of life. Indeed, vision-related quality of life is the issue of greatest importance to many cataract patients, and many lines of evidence suggest that cataract not only destroy visual function but also affect activities of daily life in many ways. ${ }^{3-7}$ It is possible that cataract surgery-induced improvements should be translated by considerable gains in patients' daily life performances and social life components.

Many studies on the impact of cataract surgery have been focused on traditional clinical tests as the primary outcome and examined patients' vision-dependent self-report questionnaires. ${ }^{6-12}$ Improvements in visual function and self-report questionnaires after
${ }^{1}$ Department of Ophthalmology, Peking University Third Hospital, Beijing, China

${ }^{2}$ Key Laboratory of Vision Loss and Restoration, Ministry of Education, Beijing, China

${ }^{3}$ Research Center of Clinical Epidemiology, Peking University Third Hospital, Beijing, China

Correspondence: W Wang, Department of Ophthalmology, Peking University Third Hospital, Number Forty-Nine North Garden Road, Hai Dian District, Beijing 100191 China

Tel: +8610822 68106; Fax: +8610822 66649 E-mail: puh3_ww@bjmu. edu.cn

Received: 7 February 2015 Accepted in revised form: 29 June 2015

Published online: 14 August 2015 
cataract surgery have been wildly confirmed. ${ }^{6-12}$ The benefit of cataract surgery, however, on vision-related ability of daily living in the elderly has not yet been investigated systemically.

To get a 'full' picture of a patient's visual improvement after cataract surgery, it is necessary to assess the objective real-life visual function in addition to clinical tests and self-reported surveys. More recently there is a coming up of trend in developing a method for evaluation of functional vision-performance-based measures (PBMs) of visual function. ${ }^{13-23}$ It involves presenting patients with commonly encountered tasks of daily life in a standardized format and getting a grade in accordance with their visual ability to complete the tasks. Pioneering studies has demonstrated that PBMs can be reliable and valid methods in assessing real-life visual ability. ${ }^{13-23}$ Owing to its significance in visual hierarchy, PBMs of visual function is becoming an important part of researches on the real-life effects of visual diseases.

Evidence suggests that visual deficiency of cataract patients could contribute to the difficulty in performing everyday tasks, such as newspaper reading and face recognition. ${ }^{13,23}$ Given the important role of functional vision of cataract patients, a new type of vision-specific PBM was developed based on Chinese population in our previous study-Real-Life Vision Test (RLVT). ${ }^{23}$ Our previous study has validated the potential efficacy of RLVT for assessing functional vision of cataract patients. It also showed that RLVT might provide information not obtainable from standard clinical measures or subjective surveys. ${ }^{23}$

This study aims to (1) enhance our understanding of the role of RLVT on assessing the actual visual ability to perform real-life tasks in patients following cataract surgery; (2) to determine how RLVT relates to both clinical measures and self-report assessment of visual function postoperatively and how they can be used together to evaluate patients' overall visual ability in everyday life. Age-related cataract patients (56) and 44 age-matched control subjects were enrolled in this study. We examined the impact of cataract surgery by three aspects of visual measures: standard clinical measures of visual function, subjective measures of visual function (self-reported surveys), and objective PBMs of visual function-the RLVT. Specifically, the changes in cataract group between the pre- and postoperative phases were analyzed. Patient-related and disease-related factors that may potentially influence their visual-related activities were also examined.

\section{Materials and methods}

The research followed the tenets of the Declaration of Helsinki Principles, informed consent was obtained from all subjects after the nature and possible consequences of the procedure had been fully explained. The study protocol was approved by an institutional review board in the Department of Ophthalmology of Peking University Third Hospital. This study was conducted in Chinese population. We certify that all applicable institutional and governmental regulations concerning the ethical use of human volunteers were followed. The study consisted of a baseline and a follow-up assessment, which were identical. The baseline assessment was administered approximately 1-2 weeks before cataract surgery, and the follow-up assessment was administered $\sim 4$ months after surgery when patients had been using their new spectacles for at least 1 month.

\section{Cataract subjects}

Subjects with bilateral uncomplicated age-related cataract (56) were selected, conforming to rigid inclusion criteria: between 60 and 82 years of age, binocular visual acuity range from 0.2 to 0.8 (logMAR), no other ocular disease other than refractive error, no history of ocular surgery and no neurological/musculoskeletal deficits. The distance spherical refractive error of both eyes ranged from $+3.00 \mathrm{D}$ to $-3.00 \mathrm{D}$ sphere, and maximum power of the cylinder was 3.00 D. Subject with low intellectual level or cognitive/depressive problems (defined as a score of 25 or lower on mini mental state examination (MMSE), ${ }^{24}$ or with a score of 41 or higher on self-rating depression scale (SDS) ${ }^{25}$ was excluded.

\section{Control subjects}

Age-matched subjects (44) with no ocular disease except for refractive error were used as controls. The same inclusion criteria of the cataract group were used, with the additional criteria that binocular visual acuity of 0.1 $\log$ MAR or better and an interocular acuity difference of no less than $0.1 \log$ MAR. Those with a stereopsis worse than $60 \mathrm{~s}$ of arc were excluded. Those with minimal nuclear sclerosis ${ }^{26}$ were eligible for inclusion because of the high prevalence of lens opacity among the people older than 70 years of age.

\section{Surgical procedures}

All cataract participants had phacoemulsification under topical and retrobulbar anesthesia through clear corneal incision. Cataract surgeries were performed by one experienced surgeon, who used identical methods for standard phacoemulsification. After surgery, all patients were treated with a combination of levofloxacin (Gravit) eye drops and prednisolone acetate (Pred Forte) ophthalmic suspension four times per day for 1 week, which were then gradually reduced. There were no intraoperative or postoperative complications in any of 
our cases in this study. The spherical mono-focal non-yellow-tinted intraocular lenses (IOLs, MatrixAcrylic 401 IOL, Medennium Inc., Irvine, CA, USA) were injected into the capsular bag. This IOL has an overall diameter of $12.5 \mathrm{~mm}$, an optic diameter of $6.0 \mathrm{~mm}$, and a refractive index of 1.56 .

\section{Clinical settings}

All participants were tested under binocular viewing conditions with habitual optical correction. Each participants was given a standard examination by ophthalmoscopy and slit-lamp biomicroscopy. A full range of clinical measures (near, intermediate, and distance visual acuity, contrast sensitivity, depth perception, and color vision test) were included. Distance visual acuities were measured using a front lit BaileyLovie letter $\log$ MAR chart (Precision Vision, La Salle, IL, USA) at a test distance of $4 \mathrm{~m}$ and scored per correct letter (0.02 logMAR per letter). ${ }^{27}$ Near and intermediate visual acuities were measured with Colenbrander mixed contrast card (Precision Vision) ${ }^{28}$ set at the distance of 40 and $100 \mathrm{~cm}$, respectively. Visual acuities were scored as the total number of letters read correctly and were converted to $\log$ MAR ( $\log 10$ minimum angle resolvable) visual acuity. Depth perception was measured using the Stereo Fly Test (Precision Vision) and results were converted into a log scale. Contrast sensitivity was measured with the OPTEC 6500P (2002 Vision Sciences Research Corp., San Ramon, CA, USA) under day-time conditions (luminance levels: $85 \mathrm{~cd} / \mathrm{m}^{2}$; spatial frequencies: $1.5,3.0,6.0,12.0$, and 18.0 c.p.d. (cycles/degree)). It was based on the Functional Acuity Contrast Test. ${ }^{29}$ All results were converted into a log scale. Color perception test was performed under manufacturer's instruction using the Farnsworth-mun-sell 100-hue (FM100-hue) test. ${ }^{30}$ The total error scores were calculated using the FM100-hue scoring software (version 3.0) with the standard method.

\section{Demographic data}

Age, gender, years of education, and the medical comorbidities were collected using a standard form. Cognitive status was assessed using the MMSE. ${ }^{24}$ The presence of depressive symptoms was assessed with the SDS. ${ }^{25}$

The reaction time (RT) was measured with a computerbased program (http://www.bbc.co.uk/science/ humanbody/sleep/sheep/reaction_version5.swf). During the test, the subjects were asked to click the mouse as soon as possible when they saw a sheep appearing on the screen. The tests were repeated 5 times and the average time was recorded as the final RT result.

\section{Self-assessed questionnaire}

Each subject completed the 25-Item National Eye Institute Visual Functioning Questionnaire (NEI-VFQ-25). ${ }^{10,31}$ The total score was calculated according to the scoring algorithm.

\section{The Real-Life Vision Test}

Each subject completed RLVT with the standard method (Table 1). In this test, Task 2 from the previous study ${ }^{23}$ Reading and Chinese character picking in reduced illumination has been deleted from RLVT due to the less prevalence of reading in the darkness in real-life situations for elder people.

\section{Reliability testing of RLVT}

In accordance with the standard assessment of test-retest stability (repeatability), RLVT testing was repeated after 2 weeks by 30 post-surgery patients and 26 control subjects. The intraclass correlation coefficient demonstrated variation in test-retest.

\section{Statistical analysis}

The Statistical Package for the Social Sciences version 18.0 (SPSS Inc., Chicago, IL, USA) was used to perform the statistical analysis. All tests of statistical significance were two-sided, and $P$-value $<0.05$ were considered statistically significant. Before the analysis, all variables were plotted and reviewed for outliers that might represent data entry errors. The distribution and relationships of all the variables were analyzed. Frequency distributions were used for categorical variables. The differences of all the variables between the cataract group and the controls were evaluated by the independent $t$-test (when the variables were normally distributed) or by the Mann-Whitney $U$-test (when the variables were not normally distributed). Paired $t$-tests were used to compare baseline and follow-up results of visual function, NEI-25 total scores and RLVT, whereas analysis of variance (when the variables were normally distributed) and the Kruskal-Wallis test (when the variables were not normally distributed) were conducted to identify the differences in groups with different types of cataract and categorical variables. Scatter plots were constructed between clinical measures, NEI-25 total scores and RLVT in order to detect the linear relationships. Bivariate relationships were evaluated using the Spearman's nonparametric correlation coefficient. Multiple linear regression analyses using stepwise selection $(P<0.05$ as the selection criterion) were used to determine which change of clinical measure(s) were significant predictors of improvements of RLVT. 
Table 1 The Real-Life Vision tasks description

Test (lighting condition) Description

Task 1. Reading and Chinese character picking (265-275 LUX)

Under the lighting of certain illumination intensity (LUX 265-275), each subject is required to pick out six specified Chinese characters from two different newspaper articles (each containing 220 characters, printed in black with gray background, in regular Chinese script (the six commonly-used characters are chosen because they involve all of the six Chinese character-making methods)). The six characters are given in the title of the test paper and each character appears only once in the article. The size of each character is $4 \mathrm{~mm} \times 4 \mathrm{~mm}$. The subject may read in silence, but must read through the articles character by character from the very beginning to the end, and mark each character that is picked out. After the test, the doctor will ask the patient about the general idea of the articles, to make sure that due attention is paid to the reading.

Task 2. Fruits and vegetables picking (320-330 LUX) Fruit and vegetable models, which are identical to real ones in size, shape, and color, are placed on a table $1 \mathrm{~m}$ away from the subjects. There is a price tag stuck to each model, the size of the words on the tags is $1 \mathrm{~cm} \times 1 \mathrm{~cm}$. Different fruits have different prices, and even the same kind of fruit have $2-3$ different prices. Subjects are given a list and are asked to pick out the right fruit/vegetable with the right price (a 2.0 Yuan peach, a 3.0 Yuan red apple, a 6.0 Yuan green apple, an 4.5 Yuan orange, a 3 Yuan yellow pepper, a 8 Yuan mango, a 5.5 Yuan yellow pear, and a 3.5 Yuan tomato) in turn and put them into a hand bag. Subjects are not allowed to touch the models before they make the decision, so as to reduce the influence of other factors.

Task 3. Button matching (145-155 LUX) Subjects are given 12 dark-colored buttons (6 pairs, similar in color and size, with an average diameter of $1 \mathrm{~cm}$ ), which are randomly put on a puce table $0.5 \mathrm{~m}$ away. The subjects are not permitted to touch the buttons before start. First, they are asked to match the 12 buttons into 6 pairs, and then thread a transparent silk string in turn through them one by one. Subjects should get a clear sight of the buttonhole before they thread the string, instead of threading it by hand-touch feeling.

Task 4. Street signs/billboard recognition This test uses the same method of face recognition mentioned below: to play slides of (1300-1350 LUX) billboards/road signs/bus stop boards (10 colored pictures taken from public places in Beijing), the size of the which vary from $8 \mathrm{~cm}$ to $20 \mathrm{~cm}$ (in height), each $1 \mathrm{~cm}$ bigger than previous one. Subjects are seated $4 \mathrm{~m}$ away from the screen, and are asked to tell the content of the billboards/street signs/bus stop boards. They can play the slides and turn the photo into a bigger size by clicking the mouse. The timing of the test is the same as that of the face recognition.

Task 5. Face recognition (1300-1350 LUX) The task is measured by presenting standardized faces of varying sizes to simulate normalsized faces at different viewing distances. Test stimuli black and white pictures, with $100 \times 100$ pixels, were selected from the Yale Face Database. All faces were cropped at the inner hairline to prevent recognition on the basis of distinctive hairline, color, or style. The pictures are played in slides and projected onto a screen $4 \mathrm{~m}$ away from the patients. Faces on the screen could be enlarged from $5 \mathrm{~cm}$ to $24 \mathrm{~cm}$ (20 gradation for each picture, the length of faces in each picture is $1 \mathrm{~cm}$ longer than the previous one). 10 pictures were selected in this test and subjects were asked one detailed question about each picture. (Picture 1 and picture 2: male or female?; picture 3 and picture 4: Chinese or foreigner?; picture 5: young or old?; picture 6: wearing a pair of glasses or not?; picture 7 to picture 10: describe the facial expression: happy, angry, scared, sad, or surprised). Before the test, we would give an elaborate explanation of the test by giving examples. After the subjects fully understood the questions, the test would begin (the timer is started). They are allowed to play the slides (gradually enlarging the pictures) by clicking the mouse by themselves if they could not see the picture clearly; if the subjects do not click the mouse after $3 \mathrm{~s}$, the slides would play the next picture automatically until they get the correct answer (the timer is stopped). Then the slides would go to the next picture until the end. No repetition of slides is allowed, participants are asked not to guess if they were unsure, and no feedback is given.

\section{Results}

\section{Demographics}

Table 2 provides a summary of the subject characteristics, clinical ophthalmic characteristics, RLVT results, and NEI-VFQ-25 total scores with respect to all independent variables. Kruskal-Wallis test revealed that there was no difference in RLVT or NEI-VFQ-25 scores due to gender or types of cataract. In cataract group, the MMSE scores was found to be significantly related to the change of face recognition $(r=-0.35, P<0.05)$, while RT was significantly related to the change of fruits and vegetables picking ( $r=0.36, P<0.05)$ and buttons matching $(r=0.37$, $P<0.01)$. The number of educational years, was 
Table 2 Participant characteristics, clinical measures of visual function, RLVT, and NEI-VFQ-25 total scores

\begin{tabular}{|c|c|c|c|c|c|c|c|c|c|}
\hline \multirow[t]{3}{*}{ Variables } & \multicolumn{4}{|c|}{ Cataract group $\mathrm{n}=56$} & \multirow{3}{*}{$\begin{array}{c}\text { P-value } \\
\text { P1 }\end{array}$} & \multicolumn{3}{|c|}{ Normal group $\mathrm{n}=44$} & \multirow{3}{*}{$\begin{array}{c}\text { P-value } \\
\text { P3 }\end{array}$} \\
\hline & \multirow{2}{*}{$\frac{\text { Preoperative }}{\text { Mean (SD) }}$} & \multicolumn{2}{|c|}{ Postoperative } & \multirow[b]{2}{*}{ Median } & & \multirow[b]{2}{*}{ Mean (SD) } & \multirow[b]{2}{*}{ Median } & \multirow[b]{2}{*}{$P 2$} & \\
\hline & & Median & Mean (SD) & & & & & & \\
\hline Age & $69.13(6.13)$ & 68.50 & $69.13(6.13)$ & 68.50 & - & $67.45(5.70)$ & 67.00 & 0.16 & 0.16 \\
\hline Educational years & $10.91(3.73)$ & 11.50 & $10.91(3.73)$ & 11.50 & - & $11.84(3.60)$ & 12.00 & 0.21 & 0.21 \\
\hline MMSE & $28.55(1.62)$ & 29.50 & $28.70(1.33)$ & 29.00 & 0.083 & $28.86(1.59)$ & 30.00 & 0.34 & 0.61 \\
\hline SDS & $35.63(3.11)$ & 36.00 & $29.54(2.52)$ & 29.50 & $<0.001$ & $28.43(3.08)$ & 29.00 & $<0.001$ & 0.05 \\
\hline RT & $0.41(0.04)$ & 0.41 & $0.40(0.04)$ & 0.39 & $<0.001$ & $0.40(0.05)$ & 0.40 & 0.64 & 0.75 \\
\hline NEI-VFQ-25 & $72.52(9.01)$ & 71.41 & $94.37(2.67)$ & 94.78 & $<0.001$ & $95.48(2.94)$ & 96.09 & $<0.001$ & 0.53 \\
\hline \multicolumn{10}{|c|}{ Distance VA (logMAR) } \\
\hline Binocular & $0.47(0.15)$ & 0.40 & $0.14(0.07)$ & 0.10 & $<0.001$ & $-0.02(0.08)$ & 0.00 & $<0.001$ & $<0.001$ \\
\hline Better eye & $0.51(0.16)$ & 0.50 & $0.19(0.08)$ & 0.20 & $<0.001$ & $0.04(0.08)$ & 0.00 & $<0.001$ & $<0.001$ \\
\hline Worse eye & $0.66(0.21)$ & 0.60 & $0.28(0.08)$ & 0.30 & $<0.001$ & $0.12(0.10)$ & 0.10 & $<0.001$ & $<0.001$ \\
\hline \multicolumn{10}{|l|}{ Near VA (logMAR) } \\
\hline Binocular & $0.51(0.13)$ & 0.50 & $0.19(0.06)$ & 0.20 & $<0.001$ & $0.11(0.08)$ & 0.10 & $<0.001$ & $<0.001$ \\
\hline Better eye & $0.56(0.13)$ & 0.50 & $0.25(0.08)$ & 0.20 & $<0.001$ & $0.16(0.08)$ & 0.20 & $<0.001$ & $<0.001$ \\
\hline Worse eye & $0.66(0.16)$ & 0.65 & $0.32(0.07)$ & 0.30 & $<0.001$ & $0.24(0.08)$ & 0.20 & $<0.001$ & $<0.001$ \\
\hline \multicolumn{10}{|c|}{ Intermediate $V A(\log M A R)$} \\
\hline Binocular & $0.53(0.12)$ & 0.50 & $0.20(0.07)$ & 0.20 & $<0.001$ & $0.03(0.10)$ & 0.00 & $<0.001$ & $<0.001$ \\
\hline Better eye & $0.56(0.12)$ & 0.60 & $0.24(0.07)$ & 0.20 & $<0.001$ & $0.10(0.08)$ & 0.10 & $<0.001$ & $<0.001$ \\
\hline Worse eye & $0.66(0.13)$ & 0.60 & $0.30(0.06)$ & 0.30 & $<0.001$ & $0.17(0.08)$ & 0.20 & $<0.001$ & $<0.001$ \\
\hline Stereopsis (log) & $2.29(0.40)$ & 2.22 & $1.90(0.18)$ & 1.90 & $<0.001$ & $1.72(0.07)$ & 1.78 & $<0.001$ & $<0.001$ \\
\hline Color vision & $143.38(33.52)$ & 139.00 & $82.75(14.56)$ & 86.00 & $<0.001$ & 42.36 (10.77) & 44.00 & $<0.001$ & $<0.001$ \\
\hline \multicolumn{10}{|c|}{ CS (log units) $\left(85 \mathrm{~cd} / \mathrm{m}^{2}\right)$} \\
\hline 1.5 c.p.d. & $1.44(0.23)$ & 1.40 & $1.70(0.15)$ & 1.70 & $<0.001$ & $1.78(0.11)$ & 1.85 & $<0.001$ & $<0.05$ \\
\hline 3.0 c.p.d. & $1.41(0.23)$ & 1.38 & $1.70(0.18)$ & 1.76 & $<0.001$ & $1.85(0.11)$ & 1.90 & $<0.001$ & $<0.001$ \\
\hline 6.0 c.p.d. & $1.24(0.67)$ & 1.36 & $1.63(0.22)$ & 1.66 & $<0.001$ & $1.77(0.11)$ & 1.81 & $<0.001$ & $<0.001$ \\
\hline 12.0 c.p.d. & $0.29(1.34)$ & 0.97 & $1.33(0.15)$ & 1.34 & $<0.001$ & $1.47(0.12)$ & 1.48 & $<0.001$ & $<0.001$ \\
\hline 18.0 c.p.d. & $-0.69(1.38)$ & -2.00 & $0.84(0.18)$ & 0.78 & $<0.001$ & $1.10(0.15)$ & 1.08 & $<0.001$ & $<0.001$ \\
\hline \multicolumn{10}{|l|}{$R L V T$} \\
\hline Task 1 & $102.18(22.04)$ & 106.29 & $53.47(9.07)$ & 54.14 & $<0.001$ & $50.43(7.01)$ & 52.19 & $<0.001$ & 0.07 \\
\hline Task 2 & $69.35(12.81)$ & 70.38 & $54.48(9.43)$ & 55.20 & $<0.001$ & $44.55(3.45)$ & 45.14 & $<0.001$ & $<0.001$ \\
\hline Task 3 & $99.34(14.76)$ & 99.67 & 58.77 (3.52) & 58.88 & $<0.001$ & $57.36(3.91)$ & 57.66 & $<0.001$ & 0.06 \\
\hline Task 4 & $44.27(6.63)$ & 44.63 & $27.75(2.43)$ & 27.74 & $<0.001$ & $27.54(3.96)$ & 26.25 & $<0.001$ & 0.06 \\
\hline Task 5 & 71.00 (15.49) & 70.34 & $33.68(3.45)$ & 33.51 & $<0.001$ & $31.92(4.58)$ & 30.64 & $<0.001$ & $<0.05$ \\
\hline
\end{tabular}

Abbreviations: c.p.d., cycles per degree; logMAR, logarithm of the minimum angle of resolution; MMSE, mini mental state examination; NEI-VFQ-25, the 25-Item National Eye Institute Visual Functioning Questionnaire; P1, P-value of the comparisons between baseline and follow-up in cataract group; P2, $P$-value of the comparisons between pre-cataract group and normal control group; P3, $P$-value of the comparisons between post-cataract group and normal control group; RLVT, real-life vision test; RT, reaction time; SDS, self-rating depression scale; task 1, reading and Chinese character-picking; task 2, fruits and vegetables picking; task3, buttons matching; task 4, street sign/billboard recognition; task 5; face recognition; VA, visual acuity.

significantly correlated with most of the changes of RLVT performance.

\section{Comparisons between cataract and control group}

The groups were not statistically significantly different with respect to age, gender distribution, years of education, RT, and MMSE scores $(P>0.05)$. However, there was a significant difference on all the clinical measures, RLVT, NEI-VFQ total scores, and the SDS scores $(P<0.01)$. As detailed in Table 2 , control subjects performed significantly better than the cataract patients in all tasks of RLVT $(P<0.01)$.

\section{Comparisons between pre- and postoperative outcomes of cataract group}

Table 2 showed that results of RLVT, clinical measures, NEI-VFQ total scores, and SDS scores were improved significantly in the cataract group from baseline to follow-up $(P<0.01)$. There was no difference between the post-surgery and normal control groups with respect to 
NEI-VFQ total scores, SDS scores, and three tasks of RLVT. However, results of clinical measures remained significantly better in the normal group than postoperative cataract patients after surgery.

\section{Relationships of postoperative changes in cataract group}

As displayed in Table 3, correlation analysis demonstrated that all the RLVT post-surgery improvements are significantly associated with most of the changes in clinical measures in cataract patients. Also, most of the changes of clinical measures had a higher correlation with the improvements of RLVT than that with the total NEI-VFQ-25 score. The strongest relationships among changes in RLVT with changes in clinical measures existed between:

- Reading and binocular near visual acuity $(r=0.56$, $P<0.001)$.

- Fruits and vegetables picking and contrast sensitivity (85 cd / $\mathrm{m}^{2} ; 1.5$ c.p.d.) $(r=0.62, P<0.001)$.

- Buttons matching and binocular near visual acuity $(r=0.65, P<0.001)$.

- Street signs recognition and better eye distance visual acuity $(r=0.51, P<0.001)$.

- Facial recognition and binocular intermediate visual acuity $(r=0.52, P<0.001)$.

Multiple regression analysis demonstrated that the significant clinical predictors of the improvements on RLVT include changes in distance, intermediate, and near visual acuity, and the binocular contrast sensitivity $\left(85 \mathrm{~cd} / \mathrm{m}^{2} ; 1.5\right.$ c.p.d.; Table 4$)$.

\section{Relationships between RLVT and NEI-VFQ-25}

Two of the RLVT subscales changes were statistically correlated to the change of NEI-VFQ total scores (Table 3). However, after adjust for the confounding factors, none of these statistically associations existed.

\section{Relationships between clinical measures and NEI- VFQ-25}

Multiple regression analysis (Table 4) showed that the changes in binocular distance visual acuity and contrast sensitivity ( $85 \mathrm{~cd} / \mathrm{m}^{2} ; 18.0$ c.p.d.) are significant clinical predictors of the change in total NEI-VFQ-25 scores.

\section{Discussion}

In this study, we found that cataract surgery was associated with improved visual function, self-report surveys, and real-life visual ability. Cataract patients experienced decreased depressive symptoms and significant improvements in the vision-targeted quality of life after surgery. Similar with other studies, $6,7,11,12,32$ patients in our study reported improved general vision, less difficulty with daily life activities and greater likelihood of engaging in social interactions following cataract surgery. Meanwhile, in comparing the changes from baseline to follow-up, cataract patients exhibited significant improvements in the RLVT. However, the

Table 3 Correlative Analysis-postoperative improvements of RLVT, Clinical measures and the NEL-VFQ-25 (SPEARMAN)

\begin{tabular}{|c|c|c|c|c|c|c|c|}
\hline & The clinical measures of visual function & \# Task 1 & \# Task 2 & \# Task 3 & \# Task 4 & \# Task 5 & $\# N E I-V F Q-25$ \\
\hline \multirow[t]{3}{*}{ \#Distance VA (logMAR) } & Binocular & $0.30^{*}$ & $0.45^{* *}$ & $0.51^{* *}$ & $0.42^{* *}$ & $0.48^{* *}$ & $0.40^{* *}$ \\
\hline & Better eye & $0.38^{* *}$ & $0.50^{* *}$ & $0.52^{* *}$ & $0.51^{* *}$ & $0.52^{* *}$ & $0.27^{*}$ \\
\hline & Worse eye & 0.15 & 0.03 & $0.27^{*}$ & 0.12 & 0.26 & $0.30^{*}$ \\
\hline \multirow[t]{3}{*}{ \#Near VA (logMAR) } & Binocular & $0.56^{* *}$ & $0.45^{* *}$ & $0.65^{* *}$ & $0.31^{* *}$ & $0.39^{* *}$ & $0.27^{*}$ \\
\hline & Better eye & $0.47^{* *}$ & $0.37^{* *}$ & $0.45^{* *}$ & 0.26 & $0.39^{* *}$ & 0.16 \\
\hline & Worse eye & 0.18 & 0.15 & $0.30^{*}$ & 0.07 & 0.23 & 0.25 \\
\hline \multirow[t]{3}{*}{ \#Intermediate VA (logMAR) } & Binocular & $0.32^{*}$ & $0.41^{* *}$ & $0.45^{* *}$ & $0.29^{*}$ & $0.52^{* *}$ & 0.71 \\
\hline & Better eye & 0.25 & $0.26^{*}$ & 0.24 & 0.16 & $0.48^{* *}$ & -0.01 \\
\hline & Worse eye & 0.20 & $0.27^{*}$ & $0.38^{* *}$ & 0.19 & $0.43^{* *}$ & 0.15 \\
\hline \# Color vision & & $0.29^{*}$ & 0.23 & 0.20 & 0.25 & 0.22 & 0.07 \\
\hline \# Stereopsis (log) & & $0.30^{*}$ & 0.14 & $0.49^{* *}$ & 0.12 & $0.30^{*}$ & $0.40^{* *}$ \\
\hline \multirow[t]{5}{*}{ \# CS (log units; $85 \mathrm{~cd} / \mathrm{m}^{2}$ ) } & 1.5 c.p.d. & $0.50^{* *}$ & $0.62^{* *}$ & $0.60^{* *}$ & $0.50^{* *}$ & $0.51^{* *}$ & $0.32 *$ \\
\hline & 3.0 c.p.d. & $0.31^{*}$ & $0.31^{* *}$ & 0.24 & 0.26 & -0.07 & -0.10 \\
\hline & 6.0 c.p.d. & 0.16 & -0.06 & 0.10 & 0.08 & 0.08 & 0.03 \\
\hline & 12.0 c.p.d. & -0.65 & -0.22 & -0.04 & -0.09 & -0.06 & 0.19 \\
\hline & 18.0 c.p.d. & -0.12 & -0.72 & -0.02 & -0.14 & 0.06 & $0.29 *$ \\
\hline \# NEI-VFQ-25 & & 0.14 & 0.26 & $0.34^{*}$ & $0.24^{*}$ & 0.26 & 1.00 \\
\hline
\end{tabular}

Abbreviations: CS, contrast sensitivity; c.p.d., cycles per degree; logMAR, logarithm of the minimum angle of resolution; NEL-VFQ-25, National Eye Institute Visual Functioning Questionnaire; RLVT, real-life vision test; task 1, reading and Chinese character-picking; task 2, fruits and vegetables picking; task 3, buttons matching; task 4, street sign/billboard recognition; task 5, face recognition; VA, visual acuity.

${ }^{*} P<0.05 ;{ }^{* *} P<0.001$; \#, postoperative improvement of each item. 
Table 4 The regression analyses of postoperative improvements of RLVT, NEI-VFQ-25, and clinical measures of visual function

\begin{tabular}{|c|c|c|c|c|c|}
\hline Dependent Variable & Independent variable & $B$ & $S E$ & BETA & P-value \\
\hline \multirow[t]{2}{*}{ \# Task 1} & Constant & 23.38 & 5.23 & & $<0.001$ \\
\hline & \# Binocular near visual acuity & 78.36 & 15.12 & 0.58 & $<0.001$ \\
\hline \multirow{3}{*}{ \# Task 2} & Constant & 5.53 & 1.68 & & 0.002 \\
\hline & \# CS $\left(85 \mathrm{~cd} / \mathrm{m}^{2}\right) 1.5$ c.p.d. & 21.36 & 4.83 & 0.50 & $<0.001$ \\
\hline & \# Binocular distant visual acuity & 11.64 & 4.83 & 0.27 & 0.019 \\
\hline \multirow[t]{4}{*}{ \# Task 3} & Constant & 12.11 & 4.32 & & 0.007 \\
\hline & \# Binocular near visual acuity & 106.77 & 22.05 & 0.95 & $<0.001$ \\
\hline & \# Better eye near visual acuity & -51.26 & 20.55 & -0.46 & 0.016 \\
\hline & \# Binocular distant visual acuity & 30.12 & 12.13 & 0.26 & 0.016 \\
\hline \multirow[t]{3}{*}{ \# Task 4} & Constant & 5.57 & 2.08 & & 0.010 \\
\hline & \# CS $\left(85 \mathrm{~cd} / \mathrm{m}^{2}\right) 1.5$ c.p.d. & 16.65 & 6.43 & 0.30 & 0.120 \\
\hline & \# Better eye distance visual acuity & 20.68 & 5.71 & 0.43 & 0.001 \\
\hline \multirow[t]{3}{*}{ \# Task 5} & Constant & 7.24 & 5.37 & & 0.183 \\
\hline & \# Binocular intermediate visual acuity & 56.02 & 18.79 & 0.39 & 0.004 \\
\hline & \# Better eye distance visual acuity & 37.14 & 14.60 & 0.33 & 0.014 \\
\hline \multirow[t]{3}{*}{ \# NEI-VFQ-25 } & Constant & 1.49 & 2.70 & & 0.584 \\
\hline & \# Binocular distance visual acuity & 22.01 & 7.88 & 0.35 & 0.007 \\
\hline & \# CS $\left(85 \mathrm{~cd} / \mathrm{m}^{2}\right) 18.0$ c.p.d. & 2.29 & 0.65 & 0.39 & 0.001 \\
\hline
\end{tabular}

Abbreviations: CS, contrast sensitivity; c.p.d., cycles per degree; logMAR, logarithm of the minimum angle of resolution; NEL-VFQ-25, National Eye Institute Visual Functioning Questionnaire; RLVT, real-life vision test; task 1, reading and Chinese character-picking; task 2, fruits and vegetables picking; task 3, buttons matching; task 4, street sign/billboard recognition; task 5, face recognition.

\#, postoperative improvement of each item.

results of two tasks in RLVT (fruits and vegetables picking and face recognition) did not reach to the 'normal level' as the control group. These findings may probably be related to the fact that spherical mono-focal nonyellow-tinted IOLs were inserted during cataract surgery and patients with mono-focal IOL may hardly adapt good intermediate visual acuity after surgery. ${ }^{33}$ In addition, our previous study suggested that intermediate visual acuity was a significant clinical predictors of both tasks (fruits and vegetables picking and face recognition), ${ }^{23}$ and this could probably explain why post-surgery patients in our study have difficulty in restoring 'normal visual-related ability' in these tasks.

All of these vision-related quality-of-life benefits are not surprising given a dramatic improvement in their visual function following cataract surgery. However, when comparing with the control group, results of post-surgery visual function were still worse than 'normal level' even at 4 months after surgery. Interestingly, these results exhibited the possibility that cataract patients could get recovered with 'normal level' of vision-related ability of daily living and self-report QoL scores at 4 months postoperatively but were not yet recovered 'clinically'. This result may be of clinical importance regarding the recovering time for real-life visual ability after cataract surgery in the elderly. Thus, RLVT may provide different information about a patient's level of actual visual ability from the standard clinical measures. Future development of RVLT should allow for more meaningful understanding in these relationships.
Although traditional clinical measures has always been the 'golden rules' for cataract surgery outcomes evaluation, $8,13,34$ we cannot deny the usefulness of performance-based and self-assessed measures of visual function. Under certain circumstances, when patients are satisfied about their visual condition after surgery (with high NEI-VFQ-25 scores) and has restored normal visual ability to perform daily activities (with normal RLVT results), it is no doubt that their quality of life will also be greatly improved, and this is of significant importance to many cataract patients. Indeed, although the changes of numbers on visual examinations may seem impressive to doctors, the restore of visual ability to recognize street signs and people's faces is more important for patients. As a result, all these three measures are unique in dealing with the different aspects of outcomes evaluation for visual function. Traditional clinical measures are the most-accepted assessments in practice. NEI-VFQ-25 acts as a valid measure of how patients feel about their ability to function subjectively, whereas RLVT is a implement measure of visual health and it measures how well cataract patients are able to perform vision-related daily activities pre- and postoperatively. The best type of evaluation should incorporate both subjective and objective measure of visual function.

In the present study, we found that visual improvement by successful cataract surgery is associated with increased functional vision, both performance based and self assessed. Similar with the previous study, ${ }^{7}$ our data revealed significant associations between improvements of RLVT and changes of clinical measures 
of visual function. RLVT was also demonstrated a stronger relationship with traditional clinical measures than NEI-VFQ-25. These findings are consistent with the notion that PBMs are more closely related to clinical assessment ${ }^{18-21}$ and less susceptible to confounding factors. ${ }^{23,35}$ All these results of current study contribute to the validity of the RLVT as a potential assessment for cataract surgery outcomes.

\section{The reliability of the RLVT}

In our previous study, ${ }^{23}$ we provided evidence of content and construct validity of RLVT. In the current study, our results offer support for the 'test-retest' reliability of the RLVT. 30 patients from the post-surgery cataract group and 26 control subjects had repeated the same RLVT tasks $\sim 2$ weeks after their previous test. The mean internal consistency coefficient of RLVT in the cataract group and control group was 0.89 and 0.88 , respectively (Table 5).

\section{Limitations}

First, the small number of subjects was the main limitation in our study. It is possible that additional relationships were not detected. Also, the subjects are from the same hospital and it may not be possible to validly generalize our findings to all individuals. Second, the exclusion criteria removed all individuals with musculoskeletal/neurologic disorders that might have influenced their testing results, and this may limit the applicability of our results to all patient subpopulations. Third, the time-instructed scoring scale still cannot fully represent the spectrum of visual damage, which could make RLVT easier to be influenced by the confounding factors other than vision. Better scoring system need to be designed in future studies. Furthermore, measuring RT in our method would also depend on patients' visual function, so the results may be influenced by this confounding factor. Further studies on establishing interrater reliability will need to be conducted before taking RLVT into consideration to use in a clinical setting.

Table 5 The intraclass correlation coefficient of RLVT in postsurgery cataract and control subjects

\begin{tabular}{ccc}
\hline RLVT & ICC of cataract group, $\mathrm{n}=30$ & ICC of normal group, $\mathrm{n}=26$ \\
\hline Task 1 & 0.93 & 0.86 \\
Task 2 & 0.92 & 0.89 \\
Task 3 & 0.85 & 0.84 \\
Task 4 & 0.84 & 0.89 \\
Task 5 & 0.90 & 0.90 \\
\hline
\end{tabular}

Abbreviations: ICC, intraclass correlation coefficient; RLVT, real-life vision test; task 1, reading and Chinese character-picking; task 2, fruits and vegetables picking; task 3, buttons matching; task 4, street sign/billboard recognition; task 5, face recognition.

\section{The potential usefulness of RLVT}

Vision-related quality of life is the issue of greatest importance to many cataract patients. The visual ability to live freely is not only an important determinant of visionrelated quality of life, but also is associated with better overall health outcomes. ${ }^{36}$ An interesting phenomenon is that, most of the patients do not come to the hospital until the disease begins to affect their ability of daily life.

Clearly, the reason that patients come to see a doctor is to get rid of the disease sufferings, and, eventually, get back to their normal life with the help of medication or surgical treatment. Therefore, as ophthalmologists, we should treat the patient as a 'whole person' who need to function in the real world but not only the disease itself. RLVT can help the doctors to examine the patient as a 'whole person' with the fact that it could measure visual disability directly by assessing what a person 'can see' in real life. In such way, RLVT may facilitate a more thorough evaluation of changes in functional outcomes and the efficacy of cataract surgery.

RLVT takes on actual performance of vision-related tasks and can be easily administered and observed by doctors/researchers. The data provided by RLVT are clearly interpretable for clinicians, especially in the context of weighing the relative benefits of cataract surgery and manage the postoperative rehabilitation. It is obvious from the clinical experiences that ophthalmologist has an important role both at the time of cataract surgery and during the decision phase preceding surgery. With the combination of traditional measures of visual function, RLVT may give a new perspective for doctors to differentiate patients who needs positive treatment from normal subjects and to advise 'right time' for patients to consider about cataract surgery.

A patient's satisfaction with surgical outcomes is highly associated with the postoperative visual ability to perform in everyday life. ${ }^{6,11}$ During post-surgery RLVT testing, many patients reported that they wished they had had surgery earlier, based primarily on their improved quality of real-life performance postoperatively. And this shows that RLVT provide an evaluation that works easier for patients than traditional clinical assessments or selfreport surveys in terms of demonstrating the real visual ability to perform daily tasks. Particularly for patients with low education background, some of them can hardly understand the meaning of visual acuity or contrast sensitivity results, whereas some of them are not able to read or understand the self-report questionnaire by themselves. Therefore, it is difficult for them to understand about their eye conditions through 'numbers' or 'words' from these standard visual examinations. As an important supplement, RLVT can be useful in telling them which aspect of vision-dependent daily life 
was affected, how well they can perform according to their current visual impairment, and also how much they can improve after cataract surgery.

In conclusion, visual outcomes of cataract surgery generally yield highly satisfactory results in real-world visual ability. Our study highlights the potential usefulness of RLVT as a meaningful adjunct to current outcomes evaluation system for cataract surgery. RLVT may effectively combine the objective nature of traditional measures of visual function with patients' actual visual ability of daily life. The use of RLVT instruments combined with clinical and self-survey methods, may be a comprehensive strategy to manifest the impact of cataract surgery on patients' overall vision-related quality of life.

\section{Summary}

\section{What was known before}

- The ultimate goal of cataract surgery is to improve the patient's visual function and, eventually, their quality of life improvements in visual function and self-report questionnaires after cataract surgery have been wildly confirmed. A new type of vision-specific performancebased measures was developed in our previous study for cataract patients-Real-Life Vision Test (RLVT). Our previous study has validated the potential usefulness of RLVT for assessing functional vision of cataract patients. RLVT might provide information not obtainable from standard clinical measures or subjective surveys.

\section{What this study adds}

- This study enhanced our understanding of the role of RLVT on assessing the actual visual ability to perform real-life tasks in patients following cataract surgery. It showed that cataract surgery could improve real-world visual ability effectively for cataract patients. The use of RLVT combined with the clinical and self-survey methods, may be the comprehensive strategy to manifest the impact of cataract surgery on patients' overall vision-related quality of life.

\section{Conflict of interest}

The authors declare no conflict of interest.

\section{Acknowledgements}

This work is supported by the grants from National Basic Research Program of China (973 Program, No.2011CB510200).

\section{References}

1 Pascolini D, Mariotti SP. Global estimates of visual impairment: 2010. Br J Ophthalmol 2011; 96: 614-618.

2 Kelly SP, Astbury NJ. Patient safety in cataract surgery. Eye 2006; 20: 275-282.
3 Dhital A, Pey T, Stanford MR. Visual loss and falls: a review. Eye (Lond) 2010; 24: 1437-1446.

4 Lundström M, Fregell G, Sjöblom A. Vision related daily life problems in patients waiting for a cataract extraction. $\mathrm{Br} \mathrm{J}$ Ophthalmol 1994; 78: 608-611.

5 Frost A, Eachus J, Sparrow J, Peters TJ, Hopper C, Davey-Smith G et al. Vision-related quality of life impairment in an elderly UK population: associations with age, sex, social class and material deprivation. Eye (Lond) 2001; 15: 739-744.

6 Chandrasekaran S, Wang JJ, Rochtchina E, Mitchell P. Change in health-related quality of life after cataract surgery in a population-based sample. Eye (Lond) 2008; 22: 479-484.

7 Owsley C, McGwin Jr G, Scilley K, Meek GC, Seker D, Dyer A. Impact of cataract surgery on health-related quality of life in nursing home residents. Br J Ophthalmol 2007; 91: 1359-1363.

8 Panchapakesan J, Rochtchina E, Mitchell P. Five-year change in visual acuity following cataract surgery in an older community: the Blue Mountains Eye Study. Eye (Lond) 2004; 18: 278-282.

9 Lundstrom M, Pesudovs K. Questionnaires for measuring cataract surgery outcomes. J Cataract Refract Surg 2011; 37: 945-959.

10 Mangione CM, Lee PP, Gutierrez PR, Spritzer K, Berry S, Hays RD. Development of the 25-item National Eye Institute Visual Function Questionnaire. Arch Ophthalmol 2001; 119: 1050-1058.

11 Lamoureux EL, Fenwick E, Pesudovs K, Tan D. The impact of cataract surgery on quality of life. Curr Opin Ophthalmol 2011; 22: 19-27.

12 Groessl EJ, Liu L, Sklar M, Tally SR, Kaplan RM, Ganiats TG. Measuring the impact of cataract surgery on generic and vision-specific quality of life. Qual Life Res 2013; 22: 1405-1414.

13 West SK, Rubin GS, Broman AT, Munoz B, Bandeen-Roche K, Turano K. How does visual impairment affect performance on tasks of everyday life? The SEE Project. Salisbury Eye Evaluation. Arch Ophthalmol 2002; 120: 774-780.

14 Owsley C, McGwin Jr G, Sloane ME, Stalvey BT, Wells J. Timed instrumental activities of daily living tasks: relationship to visual function in older adults. Optom Vis Sci 2001; 78: 350-359.

15 Szlyk JP, Seiple W, Fishman GA, Alexander KR, Grover S, Mahler CL. Perceived and actual performance of daily tasks: relationship to visual function tests in individuals with retinitis pigmentosa. Ophthalmology 2001; 108: 65-75.

16 Haymes SA, Johnston AW, Heyes AD. Relationship between vision impairment and ability to perform activities of daily living. Ophthalmic Physiol Opt 2002; 22: 79-91.

17 Owsley C, Sloane M, McGwin Jr G, Ball K. Timed instrumental activities of daily living tasks: relationship to cognitive function and everyday performance assessments in older adults. Gerontology 2002; 48: 254-265.

18 Altangerel U, Spaeth GL, Steinmann WC. Assessment of function related to vision (AFREV). Ophthalmic Epidemiol 2006; 13: 67-80.

19 Warrian KJ, Lorenzana LL, Lankaranian D, Dugar J, Wizov SS, Spaeth GL. Assessing age-related macular degeneration with the ADREV performance-based measure. Retina 2009; 29: 80-90.

20 Richman J, Lorenzana LL, Lankaranian D, Dugar J, Mayer JR, Wizov SS et al. Relationships in glaucoma patients between standard vision tests, quality of life, and ability to perform daily activities. Ophthalmic Epidemiol 2010; 17: 144-151. 
21 Warrian KJ, Lorenzana LL, Lankaranian D, Dugar J, Wizov SS, Spaeth GL. The assessment of disability related to vision performance-based measure in diabetic retinopathy. Am J Ophthalmol 2010; 149(5): 852-860.e1.

22 Charalampidou S, Nolan J, Loughman J, Stack J, Higgins G, Cassidy L et al. Psychophysical impact and optical and morphological characteristics of symptomatic non-advanced cataract. Eye 2011; 25: 1147-1154.

23 Ni W, Li X, Ao M, Zhang H, Hou Z, Si S et al. Using the reallife vision test to assess the functional vision of age-related cataract patients. Eye 2012; 26: 1402-1411.

24 Folstein MF, Folstein SE, McHugh PR. "Mini-mental state". A practical method for grading the cognitive state of patients for the clinician. J Psychiatr Res 1975; 12: 189-198.

25 Zung WW, Richards CB, Short MJ. Self-rating depression scale in an outpatient clinic. Further validation of the SDS. Arch Gen Psychiatry 1965; 13: 508-515.

26 Chylack Jr LT, Wolfe JK, Singer DM, Leske MC, Bullimore MA, Bailey IL et al. The Lens Opacities Classification System III. The Longitudinal Study of Cataract Study Group. Arch Ophthalmol 1993; 111: 831-836.

27 Bailey IL, Lovie JE. New design principles for visual acuity letter charts. Am J Optom Physiol Opt 1976; 53: 740-745.

28 Colenbrander A, Fletcher DC. Evaluation of a new mixed contrast reading card. Invest Ophtalmol Vis Sci 2004; 45: 4352-4358.

29 Hong YT, Kim SW, Kim EK, Kim TI. Contrast sensitivity measurement with 2 contrast sensitivity tests in normal eyes and eyes with cataract. J Cataract Refract Surg 2010; 36: 547-552.

30 Ao M, Chen X, Huang C, Li X, Hou Z, Zhang C et al. Color discrimination by patients with different types of lightfiltering intraocular lenses. J Cataract Refract Surg 2010; 36: 389-395.

31 Owsley C, McGwin Jr G. Depression and the 25-item National Eye Institute Visual Function Questionnaire in older adults. Ophthalmology 2004; 111: 2259-2264.

32 Finger RP, Kupitz DG, Fenwick E, Balasubramaniam B, Ramani RV, Holz FG et al. The impact of successful cataract surgery on quality of life, household income and social status in South India. PLoS One 2012; 7: e44268.

33 Zamora-Alejo KV, Moore SP, Parker DG, Ullrich K, Esterman A, Goggin M. Objective accommodation measurement of the Crystalens HD compared to monofocal intraocular lenses. J Refract Surg 2013; 29: 133-139.

34 Gomez ML. Measuring the quality of vision after cataract surgery. Curr Opin Ophthalmol 2014; 25: 3-11.

35 Friedman SM, Munoz B, Rubin GS, West SK, Bandeen-Roche K, Fried LP. Characteristics of discrepancies between selfreported visual function and measured reading speed. Salisbury Eye Evaluation Project Team. Invest Ophthalmol Vis Sci 1999; 40: 858-864.

36 Helbostad JL, Oedegaard M, Lamb SE, Delbaere K, Lord SR, Sletvold O. Change in vision, visual disability, and health after cataract surgery. Optom Vis Sci 2013; 90: 392-399. 